\title{
Fratura cominutiva e articular distal de úmero tratada com fixador esquelético externo transarticular em três gatos
}

\author{
Comminuted Articular Fractures of the Distal Humerus of the \\ Three Cats Treated by Transarticular Skeletal Fixators
}

\section{Ísis dos Santos Dal-Bó', Cássio Ricardo Auada Ferrigno', Aline Schafrum Macedo', Márcio Poletto Ferreira², Paulo Vinícius Tertuliano Marinho', Fernanda Paes ', Thales Bregadioli' \& Bianca Fiuza Monteiro'}

\begin{abstract}
Background: Humeral fractures account for $13 \%$ of all fractures in cats and commonly affect the middle diaphysis and supracondylar region with comminution. They are usually the result of high energy traumas such as falls, car accidents and firearm injuries. There are few treatment options for these types of fractures and since they are joint injuries, early surgical repair is mandatory to achieve perfect anatomical reduction, to maintain joint congruence and to allow early weight bearing. Our purpose is to report four subacute and chronic fractures of the distal diaphysis associated with comminuted supra and intercondylar fractures, stabilized with transarticular external skeletal fixator in three domestic felines.

Cases: (1)- A 3-year-old male docile Siamese cat with $3.7 \mathrm{~kg}$ of body weight was presented for consultation with a 14-day history of high rise syndrome after falling from a 12-meter high. It presented distal comminuted humeral fracture that involved the inter and supracondylar regions. It also had right carpal-ulnar subluxation and comminuted right olecranon fracture involving the articular surface in the left thoracic limb. Fracture and joint stabilization was achieved by use of a transarticular hybrid external skeletal fixator on the right front limb. Fracture healing was observed at 150 days postoperatively. (2)- A 10-year-old female aggressive mixed breed cat with $4.1 \mathrm{~kg}$ of body weight was presented for consultation with a 16-day history of high rise syndrome after falling from a 9-meter high. The cat had a comminuted right humeral articular fracture with supra and intercondylar involvement and scapular fracture on the contralateral limb. The humerus fracture was treated with a transarticular linear external skeletal fixator and fracture healing was obtained at 240 postoperative days. (3)- A 1-year-old female unsociable mixed breed cat with $4.5 \mathrm{~kg}$ of body weight was presented for consultation with a 15-day history of humeral fractures after falling from a 12-meter high building. The cat had comminuted bilateral humeral articular fracture with supra and intercondylar involvement. Fracture stabilization was achieved by use of a transarticular hybrid external skeletal fixator on both limbs. Fracture healing was achieved after 60 days on the right front limb, and after 120 days on the left front limb.

Discussion: Patients with distal humeral diaphyseal fractures should be assessed for peripheral nerve injury. None of the reported cases had neurological damage despite having suffered high energy trauma. Surgical approach to humeral distal fractures may be craniolateral, medial or caudal or even combined. In chronic fractures like those in our study, (fractures with more than 14 days of evolution), perfect anatomic reduction wasn't considered due to presence of fibrous tissue that would require excessive manipulation, leading to injuries to soft tissues and blood supply, what would increase the risk of infection and bone sequestrum. The gold standard for the treatment of articular fractures is rigid internal fixation by means of compressive screws and compressive plates, and they require open reduction. Whenever anatomical reduction can not be performed, due to either the fracture's evolution period or by the diminished bone length, closed reduction is indicated and stabilization with transarticular fixators is an alternative. Hybrid constructs are well tolerated by patients since they are lighter than circular apparatus and more versatile than linear fixators. In our study, transarticular fixators were used based on the location of the fractures and fracture period. Despite the ankyloses of the humeral-radio-ulnar joint, all patients adapted to the fixator devices very well and presented satisfactory ambulation weight bearing on the first postoperative day already. A disadvantage of the skeletal fixators is the postoperative care, which includes daily dressings, e-collaring, resting and co-operation on behalf of the owner. Two animals presented aggressive/unsocial behavior and still the prolonged postoperative management and adaptation to the fixator were successful. The results suggest that comminuted humeral distal articular fractures can be treated by the use of transarticular external skeletal fixators in cats.
\end{abstract}

Keywords: osteosynthesis, arthrodesis, elbow, feline.

Descritores: osteossíntese, artrodese, cotovelo, felino. 


\section{INTRODUÇÃO}

As fraturas umerais, em gatos, correspondem a cerca de $13 \%$ de todas as fraturas em felinos, sendo a diáfise média e a região supracondilar as mais afetadas. Geralmente são cominutivas e estão associadas a traumas de alta energia como quedas, acidentes automobilísticos e lesões por projétil de arma de fogo [8,9].

Os felinos apresentam algumas particularidades em relação à anatomia do úmero, como conformação reta, região distal ampla, ausência do forame supratroclear, presença do forame supracondilar situado proximalmente ao epicôndilo medial que alberga o nervo mediano e artéria braquial. Lateralmente, na região de terço médio da diáfise, localiza-se o nervo radial e, medialmente, os nervos mediano, musculocutâneo e ulnar $[6,8,9]$.

As opções de tratamento para fraturas cominutivas diafisárias distais associadas às fraturas supra e intercondilares são restritas. E por se tratar de lesão articular, é mandatório reparo cirúrgico mais precoce possível visando redução anatômica, manutenção da congruência articular e retorno à função $[6,8]$.

O presente trabalho tem por objetivo relatar quatro fraturas cominutivas diafisárias distais associadas às fraturas supra e intercondilar antigas, estabilizadas com fixador esquelético externo (FEE) transarticular em três felinos domésticos.

\section{CASOS}

Caso 1: Felino, Siamês, macho, 3 anos, $3,7 \mathrm{~kg}$ e temperamento dócil. Histórico de queda do quarto andar, impotência funcional dos membros torácicos direito e esquerdo (MTD e MTE) e presença de dor profunda em MTs. Os exames radiográficos torácicos revelaram fratura completa da quinta à nona costelas direitas, pneumotórax e contusão pulmonar. No MTD: fratura cominutiva diafisária distal de úmero com comprometimento inter e supracondilar e subluxação carpo-rádio-ulnar. Em MTE: fratura cominutiva de olécrano envolvendo a superfície articular. O paciente permaneceu internado por 10 dias para resolução do quadro pulmonar. Foi indicada correção das fraturas, realizada aos 14 dias de evolução. Ao exame ortopédico foi possível palpar tecido de consistência fibrosa na região das fraturas. A avaliação hematológica e as provas de função hepática não apresentaram alterações. A dosagem sérica de creatinina era de 1,9 mg/dL. No MTD foi procedida osteossíntese de úmero e estabilização carpo-rádio-ulnar com FEE híbrido transarticular composto por uma barra craniolateral com um pino de Schanz no úmero $(1,5 \mathrm{~mm})$, um anel em ferradura com um fio liso $(1,0 \mathrm{~mm})$ e um pino de Schanz $(1,0 \mathrm{~mm})$ na região diafisária média de úmero, um anel completo com dois fios lisos $(1,0 \mathrm{~mm})$ na região proximal de rádio, duas barras (lateral e medial) desde a região proximal de rádio até os metacarpianos. Na barra medial foram colocados dois pinos de Schanz e na lateral, um pino de Schanz (todos de 1,2 mm). Nos metacarpianos, foram colocados dois fios lisos (1,0 mm) [Figura 1]. Como medicações pós-operatórias (PO) foram prescritos cefalexina e tramadol. Foi aplicado meloxicam no PO imediato [1]. Foram recomendados utilização de colar elisabetano, curativos a cada sete dias, repouso e radiografias a cada 30 dias. O paciente foi capaz de se manter em estação e deambular aos três dias de PO. Os tutores optaram pelo tratamento conservador da fratura em MTE. Aos 150 dias de PO, as radiografias demonstraram consolidação de olécrano em MTE e incongruência articular. Em MTD, foi observada consolidação das fraturas. Foi indicada remoção do FEE, porém os tutores preferiram aguardar devido a alterações na função renal e ultrassonografia abdominal (insuficiência renal crônica). Os mesmos retornaram aos 330 dias de PO e o FEE foi removido. $\mathrm{O}$ paciente apresentava claudicação leve em MTE e grave em MTD.

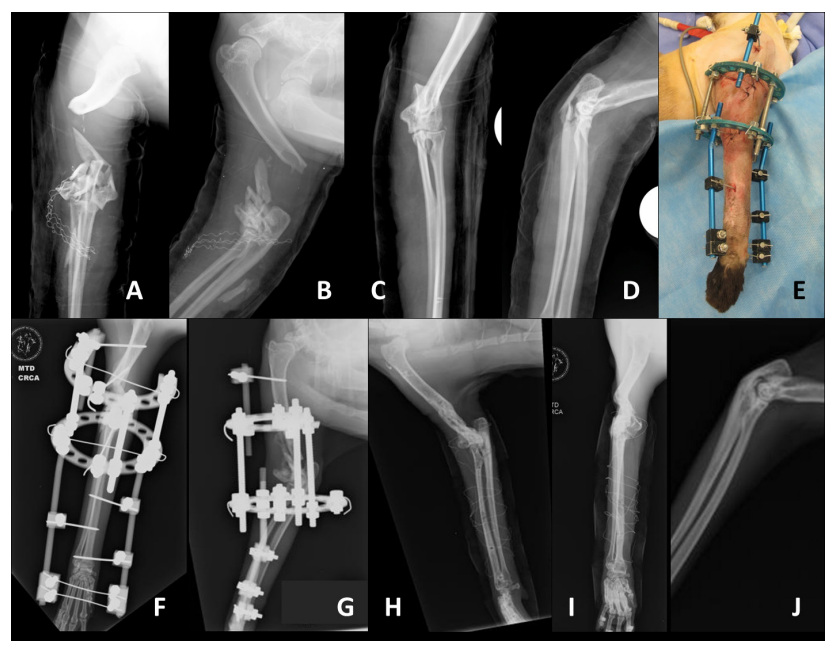

Figura 1. Imagens fotográficas do caso 1. A e B- Radiografias de úmero direito logo após o trauma revelando fratura cominutiva distal de úmero com comprometimento supra e intercondilar. C e D- Radiografias da articulação URU esquerda evidenciando fratura cominutiva de olécrano envolvendo a superfície articular. E- Aspecto do FEE híbrido transarticular no PO mediato. F e G- Radiografias de MTD no PO mediato. H e I- Radiografias de MTD aos 330 dias de PO, após remoção do FEE demonstrando consolidação da fratura e remodelamento ósseo. J- Radiografia da articulação URU aos 150 dias de tratamento conservador. 
Caso 2: Felino, SRD, fêmea, 10 anos, 4,1 kg e temperamento agressivo. Histórico de queda do terceiro andar de um prédio havia sete dias apresentando impotência funcional de MTD. Foi levada ao veterinário que recomendou amputação do membro devido à gravidade da fratura. Ao exame ortopédico observou-se presença de dor profunda, aumento de volume de consistência fibrosa, mobilidade, crepitação e dor nas regiões diafisária distal de úmero direito e proximal de úmero esquerdo. A avaliação radiológica revelou fratura cominutiva de úmero com perda da relação articular e comprometimento supra e intercondilar em MTD e fratura de colo da escápula em MTE. As radiografias de tórax, eletrocardiograma, hemograma e provas de função renal e hepática não apresentaram alterações. Optou-se pela redução fechada da fratura, aos 16 dias de evolução, com auxílio de fluoroscopia e osteossíntese com FEE linear transarticular composto por uma barra craniomedial (com dois pinos proximais craniais, dois pinos na porção medial do côndilo umeral e um pino no olécrano), uma barra lateral (com 2 pinos proximais e um pino no olécrano) e uma barra lateral no rádio (com 2 pinos). Foi colocada uma barra conectora entre as barras craniomedial e lateral do úmero na sua porção proximal. Foram utilizados somente pinos de Schanz (1,0, 1,2 e 1,5 mm) [Figura 2]. Não foi realizada osteossíntese da escápula MTE devido ao bom apoio do membro. Como medicações PO foram prescritos cefovecina, dipirona, tramadol e meloxicam [1]. Foram recomendados os mesmos cuidados PO do caso 1. No primeiro dia PO, a paciente foi capaz de utilizar o MTD apresentando claudicação grave. Aos 60 dias de PO foi observada claudicação moderada e foi removida a barra lateral do rádio do FEE. Aos 150 dias PO, foi observada contratura e atrofia musculares leves em região de tríceps e os proprietários relataram que quando o animal deitava permanecia com a articulação úmero-rádio-ulnar (URU) estendida e com a articulação escápulo-umeral (EU) flexionada. A paciente adotava essa postura durante a deambulação de forma intermitente. Então, a barra conectora e craniomedial foram removidas e recomendada fisioterapia. Aos 240 dias PO foi identificada consolidação da fratura e aos 270 dias PO foi removido o restante do FEE. Aos 360 dias de PO observou-se remodelação óssea, claudicação moderada devido à anquilose da articulação URU. $\mathrm{O}$ animal era capaz de subir e descer dos móveis. A paciente recebeu alta.

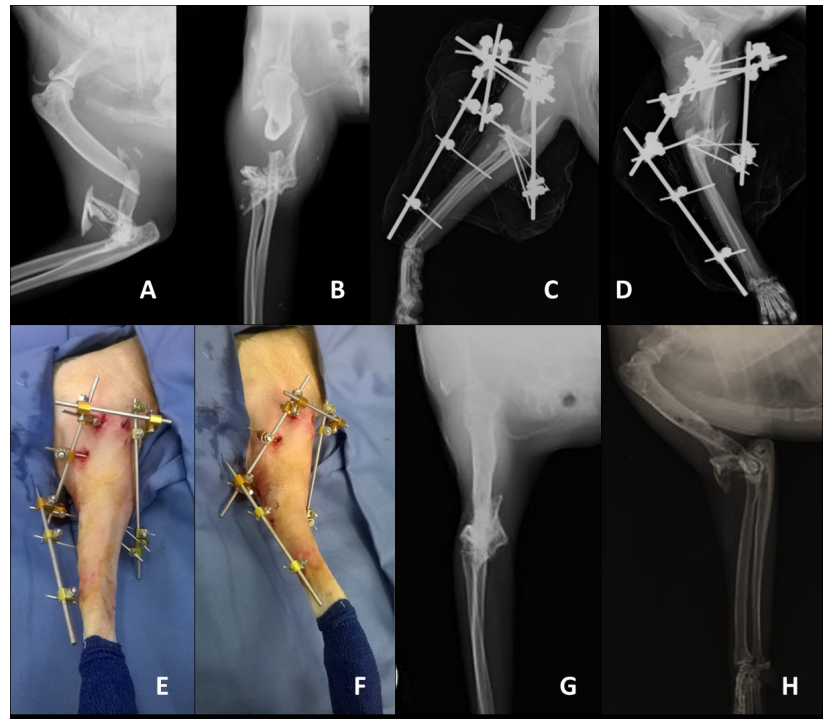

Figura 2. Imagens fotográficas do caso 2. A e B- Radiografias préoperatórias de úmero direito, com sete dias de evolução, evidenciando fratura cominutiva distal de úmero com comprometimento supra e intercondilar. C e D- Radiografias do PO imediato demonstrando FEE linear transarticular. E e F- Aspecto do FEE no PO imediato. G e H- Radiografias aos 270 dias de PO.

Caso 3: Felino, SRD, fêmea, 1 ano, $4,5 \mathrm{~kg}$ e temperamento arredio. Histórico de queda do quarto andar de um prédio e impotência funcional dos MTD e MTE. A paciente permaneceu internada por 10 dias após o trauma para tratamento de pneumotórax e contusão pulmonar. Após a resolução do quadro pulmonar, foi indicada correção das fraturas, realizada aos 15 dias de evolução. Ao exame ortopédico observou-se presença de dor profunda, aumento de volume de consistência rígida, pouca mobilidade e dor em região diafisária distal de úmero em MTD e MTE. As radiografias evidenciaram ausência de contusão pulmonar/pneumotórax e integridade diafragmática, fratura cominutiva de úmero com perda da relação articular e comprometimento supra e intercondilar em ambos os membros torácicos. Os exames pré-operatórios não apresentaram alterações. Foi realizada redução fechada e aplicação de FEE híbrido transarticular no MTD composto por uma barra craniolateral no úmero (com dois pinos de Schanz de 1,5 mm), um semi-anel na região distal de úmero (com dois fios olivados de 1,2 mm), um anel completo na região proximal de rádio (com dois fios olivados de 1,2 mm), uma barra lateral e outra medial no rádio com dois e um pino de Schanz (1,2 mm), respectivamente. As barras do rádio foram retorcidas a fim de proporcionar a angulação de, aproximadamente, $135^{\circ}$ da articulação URU. No MTE foi aplicado FEE híbrido transarticular composto por 
duas barras no úmero, uma craniolateral (com 2 pinos de Schanz de 1,5 mm) e uma lateral (com 1 pino de Schanz de $1,5 \mathrm{~mm}$ ), um quarto de anel na região distal craniolateral de úmero (com dois pinos de Schanz de 1,2 $\mathrm{mm}$ ) e uma barras lateral e craniomedial no rádio com dois e um pino de Schanz de 1,2 mm, respectivamente (Figura 3). O manejo PO foi o mesmo dos pacientes dos casos anteriores e foram prescritos amoxicilina e ácido clavulânico, tramadol, dipirona, meloxicam e omeprazol [1]. No primeiro dia PO observou-se claudicação moderada em MTD e claudicação grave intermitente/impotência funcional e apoio sobre o dorso dos dígitos em MTE. Foi realizada imobilização na região de carpo e dígitos para facilitar o apoio. Aos três dias PO, a imobilização foi removida e a paciente apresentava claudicação moderada bilateral. Aos 14 dias PO, a paciente era capaz de brincar com uma bolinha utilizando o MTD para movimentar o objeto. Nas radiografias de 60 dias PO, foi observada consolidação da fratura do MTD e fratura do MTE em evolução de consolidação. No mesmo período, a paciente apresentava claudicação moderada bilateral e, em alguns momentos elevava ambos os MTs. O FEE do MTD foi removido aos 110 dias de evolução. Aos 120 dias de PO, foi observada consolidação da fratura do MTE. Aos 150 dias de PO, o FEE do MTE foi removido. Sem os FEEs, o animal deambulava satisfatoriamente, sendo capaz de pular, subir e descer de móveis e escadas, brincar com objetos e utilizar arranhadores. Aos 240 dias de PO, foram realizadas as últimas radiografias, onde foram observados remodelamento das fraturas, preenchimento dos orifícios feitos pelos pinos dos FEE e anquilose das articulações URU (Figura 4).

\section{DISCUSSÃO}

As fraturas envolvendo a porção distal do úmero são comuns em cães devido ao aspecto delgado da diáfise distal e à presença do forame supratroclear. Nos gatos, devido às características anatômicas umerais, as fraturas articulares ocorrem em traumas de alta energia $[2,6,8]$. Nesse tipo de trauma, em geral, o paciente apresenta lesões concomitantes em tórax, abdômen e sistema nervoso [5,7]. As quatro fraturas estudadas envolviam a região supracondilar e articular e dois pacientes apresentavam comprometimento pulmonar concomitante. No caso 2 , foi realizado tratamento cirúrgico uma vez que os tutores decidiram procurar uma segunda opinião, já que foi indicada amputação do membro no seu primeiro atendimento, procedimento indicado para esses casos até a década de 1990 [5].

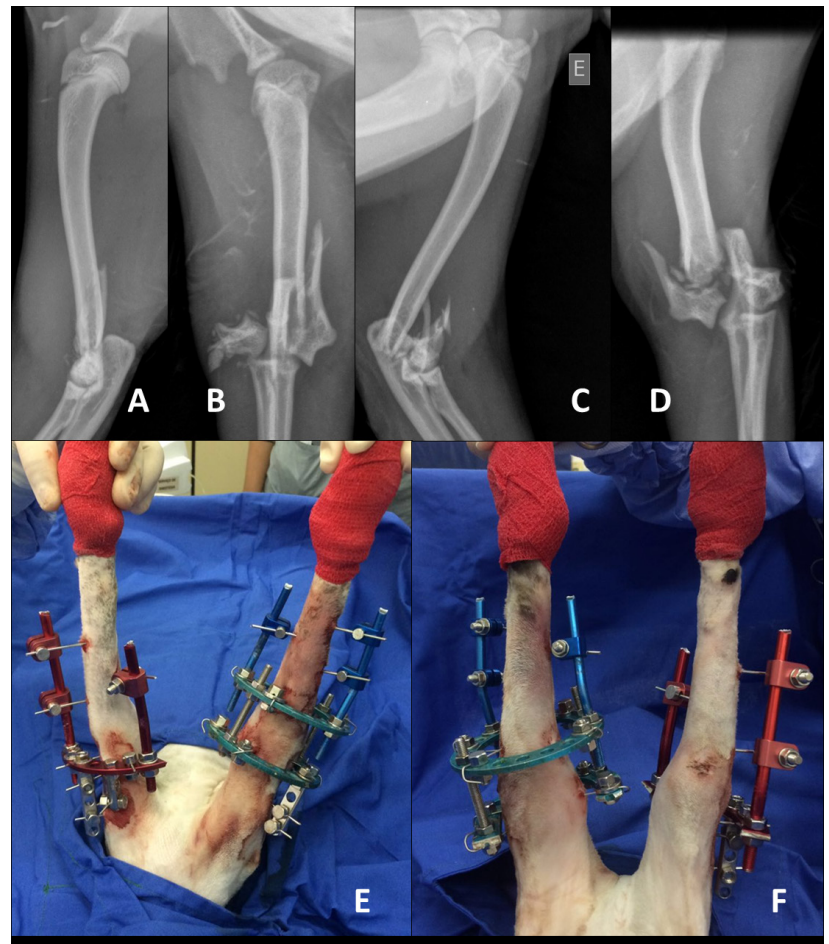

Figura 3. Imagens fotográficas do caso 3. A e B- Radiografias de úmero direito. $\mathrm{C}$ e D- Radiografias de úmero esquerdo. As quatro imagens foram obtidas aos 10 dias após o trauma e demonstram fraturas cominutivas distais de úmero com comprometimento supra e intercondilar. E e F- Aspecto dos FEE híbridos transarticulares no PO imediato nas vistas cranial e caudal, respectivamente. FEE vermelho: MTE e FEE azul: MTD.

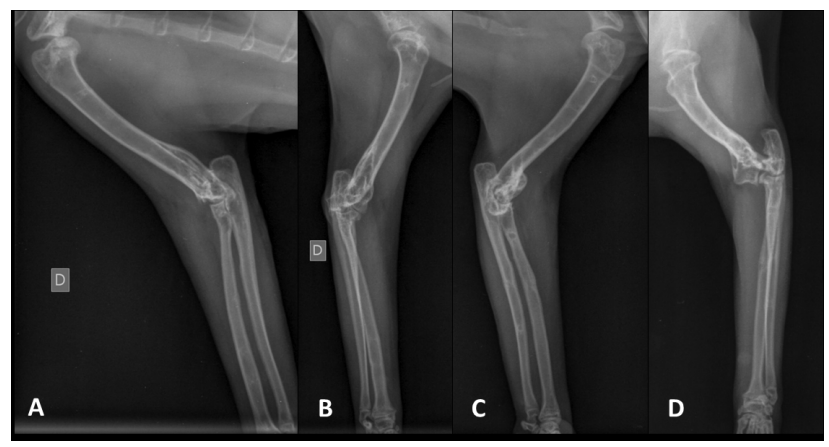

Figura 4. Imagens fotográficas do caso 3. Radiografias de úmero, articulação URU, rádio e ulna dos MTD e E aos 240 dias de PO, onde observam-se remodelamento das fraturas, preenchimento dos orifícios feitos pelos pinos dos FEE e anquilose das articulações URU. A e B- MTD nas projeções ML e CrCa, nesta ordem. C e D- MTE nas projeções ML e CrCa, respectivamente.

Os pacientes que apresentam fraturas diafisárias distais de úmero devem ser avaliados quanto à lesão de nervo periférico [7,8]. Os casos relatados não presentavam lesão neurológica apesar do trauma de alta energia, contudo foi observado comprometimento de ambos os MTs em diferentes localizações. 
A abordagem às fraturas distais de úmero pode ser craniolateral, medial ou caudal ou ainda combinação destas [6]. Em casos como os desse trabalho, em que as fraturas articulares foram consideradas antigas, apresentado 14, 16 e 15 de evolução e havia presença de tecido fibroso, a redução aberta necessita de excessiva manipulação, levando à lesão aos tecidos moles e suprimento sanguíneo, aumentando risco de infecção e formação sequestro ósseo.

O padrão-ouro para tratamento das fraturas articulares é a fixação interna rígida por meio de parafusos com efeito compressivo e placas, os quais necessitam de redução aberta [5,6]. Em casos em que a redução anatômica não pode ser realizada, seja pelo tempo de evolução da fratura ou pela ausência de comprimento ósseo capaz de suportar os parafusos, a redução fechada é indicada e a estabilização com FEE transarticulares é uma alternativa [3,5].

Os FEE são amplamente utilizados em cães e gatos, podendo ser empregados em fraturas abertas ou fechadas, por meio de redução aberta ou fechada. Promovem mínimo trauma tecidual durante sua aplicação e podem ser associados a outros implantes e sofrer ajustes no PO. Os FEE lineares são versáteis permitindo montagem e desmontagem simples e os circulares são usados para tratamento de fraturas, não-união e osteomielite bem como alongamento do membro e correção de deformidades angulares [4].

Os FEE híbridos reúnem características dos FEE lineares e circulares. As vantagens dos componentes circulares são utilização do anel em pequenos fragmentos próximos às articulações a fim de promover micromovimentos durante o apoio do membro acelerando a formação de calo ósseo. Os componentes lineares, contudo, são empregados no fragmento ósseo mais longo. São utilizadas diversas configurações de acordo com a rigidez que se deseja e o osso fraturado [4,8]. Os FEE híbridos são bem tolerados pelos pacientes, pois são mais leves em comparação aos circulares e mais versáteis que os lineares. No presente estudo, entretanto, utilizou-se FEE transarticulares em função da localização das fraturas e do seu tempo de evolução. Apesar da fixação da articulação URU, os pacientes se adaptaram muito bem os dispositivos apresentando deambulação satisfatória já no primeiro dia PO. Uma desvantagem dos FEE é o cuidado PO, que inclui curativos, utilização de colar, repouso e cooperação por parte do tutor [4]. Dois animais apresentavam comportamento agressivo/arredio, o que não impediu o manejo prolongado de PO e adaptação ao fixador.
No caso 2, a aplicação do FEE foi realizada com auxílio de fluoroscopia, o que facilitou a inserção do primeiro pino de Schanz na região do côndilo umeral. Esse método assim como a redução fechada, tração intra-operatória e pré-montagem do FEE colaboram para diminuir o tempo cirúrgico e lesão vascular uma vez que se tratava de um paciente idoso [4].

Foi realizado FEE linear transarticular no caso 2 em função do tipo de fratura e pela impossibilidade de aplicação de FEE linear tipo II ou III, os quais promovem maior estabilidade. Em alternativa a essas montagens em fêmur e úmero devido à sua proximidade com o abdômen e tórax, respectivamente, há a configuração tie-in, ou seja a associação de um pino intramedular que é conectado ao FEE tipo I, a adição de uma barra ou a aplicação de FEE transarticular [3,5]. Entretanto, assim como nos demais casos, poderia ter sido aplicado FEE híbrido transarticular.

Nas três fraturas tratadas com FEE híbrido transarticular foi observada maior facilidade de aplicação do mesmo em relação ao caso 2 , uma vez que a associação de componentes circulares e lineares permite maior liberdade no posicionamento dos pinos [4]. Para a estabilização das fraturas do caso 3, foi favorecida pela versatilidade do FEE híbrido porque a paciente apresentava fratura bilateral e dispositivos iguais poderiam prejudicar a deambulação.

Um estudo que avaliou 130 casos de fraturas de úmero em 23 gatos e 107 cães relatou diversas técnicas de osteossíntese sendo placa e parafuso a mais usada e observou pior prognóstico nas fraturas distais que envolviam a articulação devido à falha nos implantes e a erros técnicos como falha na banda de tensão do olécrano, redução não anatômica e parafusos na fossa do olécrano [2]. A única complicação observada nas quatro fraturas relatadas aqui foi o desenvolvimento de contratura leve na região de tríceps no caso 2 , a qual foi resolvida com fisioterapia. Nem mesmo presença de secreção ou crostas [5] foi identificada ao longo do PO prolongado desses casos.

Há autores que condenam o uso de FEE transarticulares porque esse método pode levar à restrição da amplitude de movimento articular após sua remoção [6]. Nos casos relatados, entretanto, já havia restrição de movimento, pois as fraturas eram antigas e o que se buscou foi estabilizar o úmero para que os pacientes pudessem deambular satisfatoriamente. Tal limitação não os impediu de deambular, correr, subir e descer de escadas e móveis e brincar com objetos. 
I.S. Dal-Bó, C.R.A. Ferrigno, A.S. Macedo, et al. 2017. Fratura cominutiva e articular distal de úmero tratada com

Os resultados dessa série de casos sugerem que as fraturas cominutivas distais de úmero com comprometimento articular podem ser tratadas com o emprego de FEE transarticular em gatos.
Acknowledgements. À M.V. Vanessa C.M. Ferraz pela disponibilização de ferramentas de diagnóstico por imagem para o presente relato. Declaration of interest. The authors report no conflicts of interest. The authors alone are responsible for the content and writing of the paper.

\section{REFERENCES}

1 Andrade S.F. 2008. Manual de Terapêutica Veterinária. 3.ed. São Paulo: Roca, 936 p.

2 Bardet J.F., Hohn R.B., Rudy R.L. \& Olmstead M.L. 1983. Fractures of the humerus in dogs and cats - a restrospective study of 130 cases. Veterinary Surgery. 12(2): 73-77.

3 Beck J.A. \& Simpson D.J. 1999. Type 1-2 hybrid external fixator with tied-in intramedullary pin for treating comminuted distal humeral fractures in a dog and a cat. Australian Veterinary Journal. 77(1): 18-20.

4 Jiménez-Heras M., Rovest G.L., Nocco G., Barilli M., Bogoni P., Salas-Herreros E., Armato M., Collivignarelli F., Vegni F. \&Rodrígues-Quiros J. 2014. Evaluation of sixty-eight cases of fracture stabilisation by external hybrid fixation and a proposal for hybrid construction classification. BMC Veterinary Research. 10: 189.

5 Langley-Hobbs S.J., Carmichael S. \& McCartney W.T. 1997. External skeletal fixation for stabilization of comminuted humeral fractures in cats. Journal of Small Animal Practice. 38(7): 280-285.

6 Macias C., Gibbons S.E. \&Mckee W.M. 2006. Y-T humeral fractures with supracondilar comminution in five cats. Journal of Small Animal Practice. 47(2): 89-93.

7 Moses P.A., Lewis D.D., Lanz O.I., Stubbs W.P., Cross A.R. \& Smith K.R. 2002. Intramedullary interlocking nail stabilization of 21 humeral fractures in 19 dogs and one cat. Australian Veterinary Journal. 80(6): 336-343.

8 Silva H.R., Clements D.N., Yeadon R. \& Fitzpatrick N. 2012. Linear-circular external skeletal fixation of intracondylar humeral fractures with supracondylar comminution in four cats. Veterinary and Comparative Orthopaedics and Taumatology. 25(1): 61-66.

9 Voss K., Langley-Hobbs S.J. \& Montavon P.M. 2009. Humerus. In: Montavon P.M., Voss K. \& Langley-Hobbs S.J. (Eds). Feline orthopedic surgery and musculoskeletal disease. London: Saunders Ltd., pp.343-358. 